\title{
Die Entstehung von Savannen - Gräser und Huftiere im Wettstreit
}

\author{
Georg Zizka \& Karen Hahn
}

\begin{abstract}
Savannas are typified by a relatively closed cover of grasses. Many of these are so-called $\mathrm{C}_{4}$ plants. Animals, plants, humans and fire have affected and molded the landscape over millions of years. The interplay of herbivores and grasses in the savanna is a prominent example of coevolution: the grasses deposit large amounts of crystalline silica in their leaves as a feeding deterrent, while the large mammalian herbivores have high-crowned teeth, which helps to counteract abrasion caused by such crystals.
\end{abstract}

\section{Zusammenfassung}

Eine gut entwickelte Grasschicht ist ein typisches Merkmal von Savannen. Viele Gräser der Savanne sind sogenannte $\mathrm{C}_{4}$-Pflanzen. Der Einfluss von Tieren, Menschen oder Feuer hat die „Architektur“ der Savanne über viele Millionen Jahre hinweg geprägt. Pflanzenfresser sowie Gräser der Savanne sind klassische Beispiele für Coevolution. Die gegenseitige Anpassung zeigt sich z.B. in der Einlagerung von Kieselsäure in die Blätter (als Fraßschutz) und Ausprägung von hochkronigen Zähnen bei den Tieren (gegen zu schnelle Abnutzung).

\section{Einführung}

Savannen sind von der Vegetation her charakterisierte, vielfältige und durch Klima, Boden, Wechselwirkungen mit der Tierwelt sowie menschlichem Einfluss geprägte Lebensräume. Der Lebensraum Savanne erstreckt sich über rund 33 Millionen $\mathrm{km}^{2}$, das ist rund ein Fünftel der Festlandsfläche (BeErling \& Osborne 2006). Savannen präsentieren sich als bemerkenswert vielfältig und wandeln sich oft kontinuierlich entlang eines Klimagradienten, so z.B. in Westafrika. Außerordentlich schwierig ist es, die Entstehung der Savannen in der Erd- geschichte $\mathrm{zu}$ rekonstruieren. Informationen dazu bieten Fossilien, auch deren chemische Zusammensetzung, mit der sich Vorkommen und Ausbreitung charakteristischer Savannengräser rekonstruieren lassen. Besonders spannend in Bezug auf die afrikanischen Savannen ist die Frage, wie Gräser und die sich davon ernährenden Huftiere sich aneinander ange-

Abb. 1: Verschiedenste Faktoren beeinflussen die Ausprägung der Savanne: Vom Klima her können Savannen mit Bäumen und Sträuchern vorkommen (im Hintergrund). Im Vordergrund ist der Boden betonhart (sog. Bowal), hier können nur Gräser gedeihen.

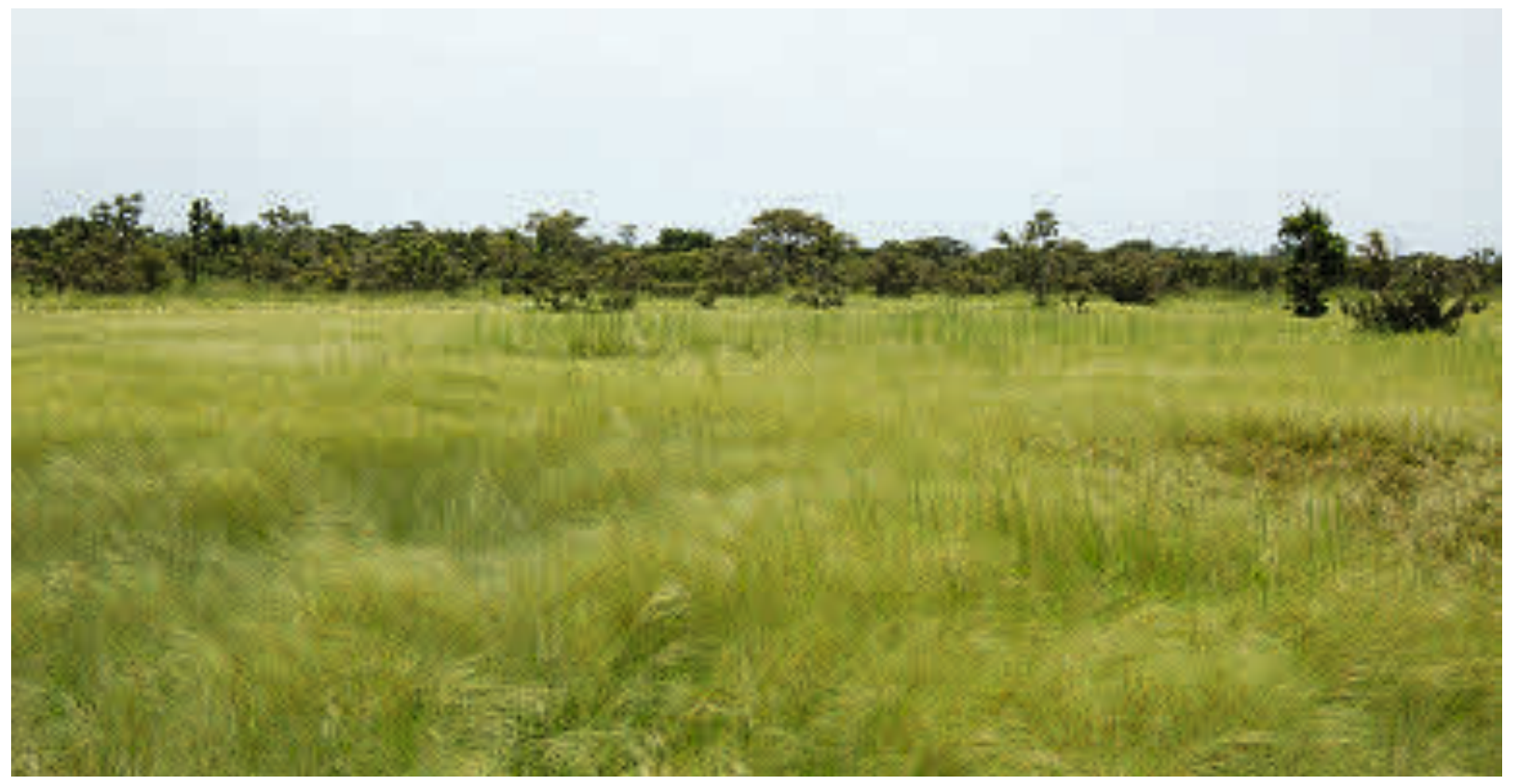




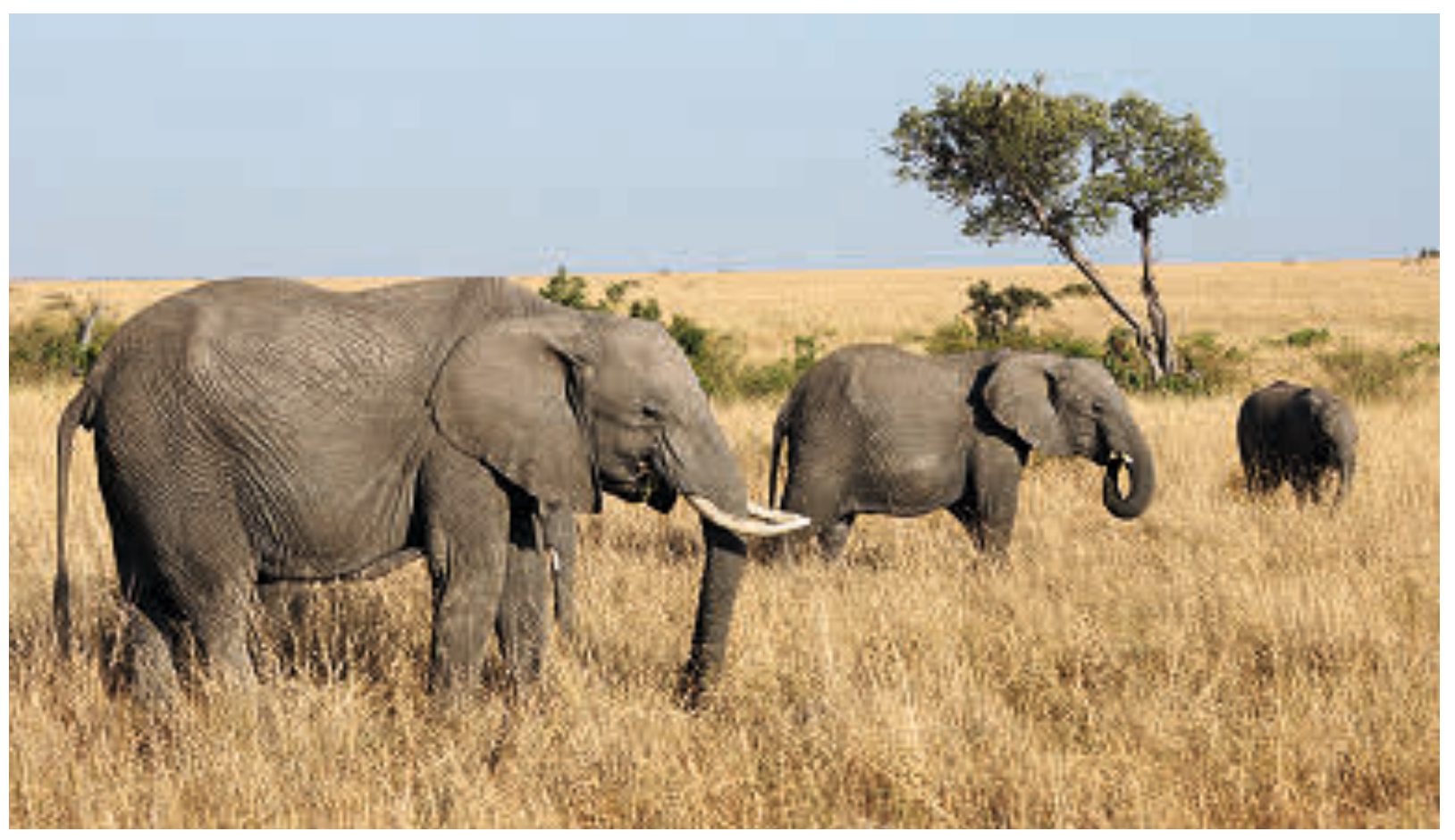

passt und damit die heutigen Savannen geformt haben.

\section{Wann sind Savannen entstanden?}

Auch wenn der Fossilbefund insgesamt lückenhaft ist, so ist bekannt, dass sich die Savannen im Känozoikum, also innerhalb der letzten 66 Millionen Jahre, entwickelt haben. Eine gut ausgebildete Grasschicht ist ein wichtiges Merkmal der Savanne. Gräser (Pflanzenfamilie Poaceae) sind erst seit der späten Kreidezeit (vor ca. 70 Mio. Jahren) fossil nachgewiesen, Savannen können also kaum viel früher entstanden sein. Ebenfalls aus der späten Kreidezeit sind auch heute ausgestorbene Säugetiere belegt, deren Zahnformen auf Gräser als Hauptnahrung hindeuten. Nach neueren Rekonstruktionen breiteten sich im mittleren und späten Känozoikum waldfreie, offene Lebensräume aus, Wälder dagegen gingen zurück. Wahrscheinlich geschah dies aufgrund globaler Klimaveränderungen (kühler, trockener, stärkere Saisonalität). Die waldfreien Lebensräume wurden aber noch von den stammesgeschichtlich älteren sog. $\mathrm{C}_{3}$-Gräsern dominiert (s.u.), die in den heutigen Savannen selten sind. Erst wesentlich später, vor 8-3 Mio. Jahren (spätes Neogen) erfolgte dann die rasante Ausbreitung der be- sonders angepassten $\mathrm{C}_{4}$-Gräser. Diese erlangten ihre beherrschende Rolle in tropischen waldfreien Habitaten (EDwards et al. 2010) wie z. B. in Savannen. Die Entstehung dieser durch ihren Stoffwechsel charakterisierten Grasgruppe liegt aber schon wesentlich länger zurück (ca. 30 Mio. Jahre). Ursache für ihre Entwicklung war das damalige Absinken des $\mathrm{CO}_{2}$-Gehaltes in der Atmosphäre. Unklar ist allerdings, warum zwischen dem ersten Auftreten der $\mathrm{C}_{4}$ Gräser und ihrer erfolgreichen weltweiten Ausbreitung über 20 Mio. Jahre verstrichen. Eine Interpretation nimmt als Ursache für die Ausbreitung der $\mathrm{C}_{4}$-Gräser das spätere Entstehen offener Lebensräume unter warm-trockenem Klima an, in denen diese Gräser besonders konkurrenzfähig sind (STRÖMBERG 2011).

Die erfolgreiche Ausbreitung der Gräser und der von ihnen geprägten offenen Vegetationstypen war eine der tiefgreifenden Veränderungen

Abb. 2 (oben): Auch Elefanten sind wichtige „Architekten“ der Savanne.

Abb. 3 (Seite 63): Savannengräser sind eine wichtige Nahrungsgrundlage für viele Tiere wie z. B. für diese Antilopen. 


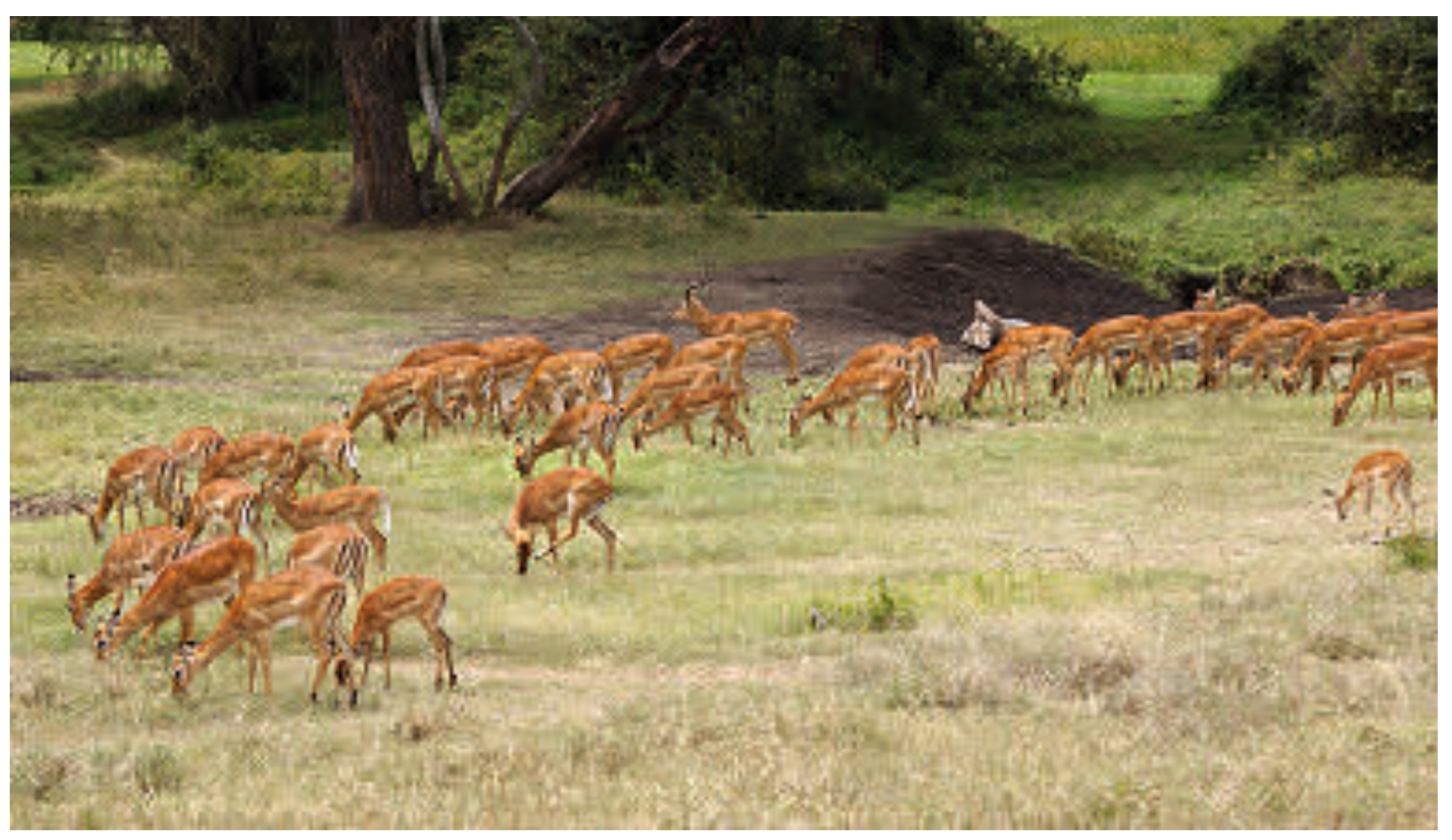

in der Erdgeschichte. Sie beeinflusste das globale Klima und war Voraussetzung für die Evolution einiger Tiergruppen (STRÖMBERG 2011). Anzumerken ist, dass zwischen den Kontinenten z.T. erhebliche Unterschiede in zeitlichem Ablauf und Umfang der Ausbreitung offener, grasreicher Lebensräume bestehen.

\section{Was sind $\mathrm{C}_{3}$ - und $\mathrm{C}_{4}$-Gräser?}

Beide Gruppen gehören zur Pflanzenfamilie der Süßgräser (Poaceae), die rund 11000 Arten umfasst, darunter die für unsere Ernährung so wichtigen Getreidegräser. Sie unterscheiden sich darin, wie das Kohlendioxid $\left(\mathrm{CO}_{2}\right)$ aus der Luft gebunden wird. Die Bezeichnung $\mathrm{C}_{3}$ - und $\mathrm{C}_{4}$-Gräser nimmt auf das Stoffwechselprodukt Bezug (chemisches Zeichen für Kohlenstoff: C; $\mathrm{C}_{3} / \mathrm{C}_{4}$ : Verbindung mit 3 oder 4 Kohlenstoffatomen), das bei der Bindung von atmosphärischem $\mathrm{CO}_{2}$ entsteht. $\mathrm{C}_{4}$-Gräser nutzen dieselben Stoffwechselwege zur $\mathrm{CO}_{2}$-Assimilation wie die $\mathrm{C}_{3}$-Gräser, haben jedoch einen zusätzlichen, effektiven Schritt der $\mathrm{CO}_{2}$-Fixierung vorgeschaltet und diesen im Blatt räumlich von der nachfolgenden $\mathrm{CO}_{2-}$ Verarbeitung getrennt. Dadurch können sie bei geringerer $\mathrm{CO}_{2}$-Konzentration in der Atmosphäre, hohen Temperaturen, hoher Lichtintensität und Trockenheit wesentlich produktiver Photosynthese betreiben als die $\mathrm{C}_{3}$-Gräser und dominieren daher in der Savanne. Nachteil des $\mathrm{C}_{4}$-Stoffwechsels ist aber ein etwas höherer Energieaufwand pro fixiertem Molekül $\mathrm{CO}_{2}$. Dadurch erreichen $\mathrm{C}_{4}$-Gräser erst bei höheren Lichtintensitäten eine Netto-Stoffproduktion als $\mathrm{C}_{3}$-Gräser, $d$. h. sie können bei geringen Lichtintensitäten „verhungern“. In den gemäßigten Breiten (also bei uns) oder schattigen Waldhabitaten dominieren daher die $\mathrm{C}_{3}$-Gräser.

Für die Rekonstruktion der Geschichte der Savanne ist das Vorkommen dieser beiden Stoffwechselwege entscheidend. Da für die $\mathrm{CO}_{2}$-Fixierung aus der Luft zwei verschiedene Enzyme verantwortlich sind, werden die in der Natur vorkommenden natürlichen Kohlenstoffisotope ${ }^{12} \mathrm{C}$ und ${ }^{13} \mathrm{C}$ in unterschiedlichem Verhältnis aufgenommen. Dieses Verhältnis der Isotope kann man noch nach Jahrmillionen an Gestein oder Fossilien untersuchen und so heute feststellen, ob sich eine Tierart vor ihrem Tod überwiegend von $\mathrm{C}_{3}$ - oder $\mathrm{C}_{4}$-Gräsern ernährt hat.

Der $\mathrm{C}_{4}$-Stoffwechsel soll für etwa $30 \%$ der weltweiten Photosyntheseproduktion verantwortlich sein, findet sich aber nur bei etwa 3\% der Blütenpflanzen. Darunter sind vor allem Gräser (rund 4500 Arten, etwa $60 \%$ der Süß- 

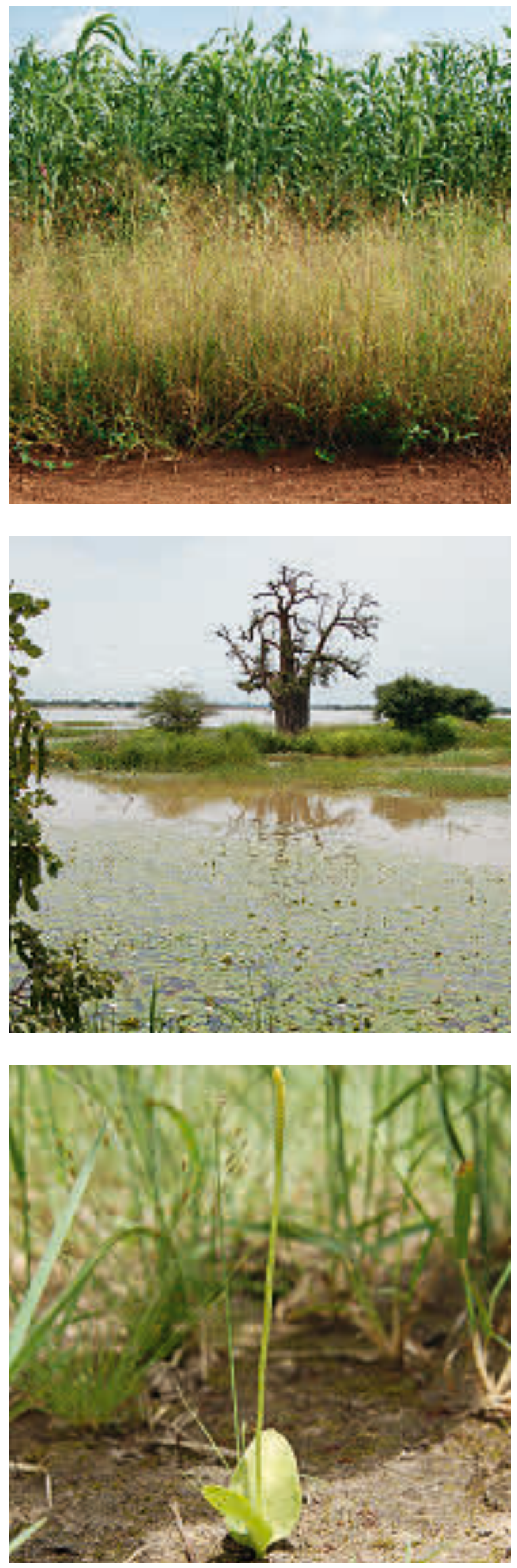

gräser, Bouchenak-Khelladi \& Hodkinson 2011) und einige andere Arten aus einer Reihe weiterer Familien. Alle anderen Blütenpflanzen, darunter alle Gehölze der Savanne, sind $\mathrm{C}_{3}-$ Pflanzen.

\section{Welche Rolle spielten natürliche Feuer?}

Feuer haben wahrscheinlich von Anfang an eine wichtige Rolle bei der Entstehung der Savannen gespielt. Sie entstehen natürlicherweise durch Blitzschlag und wirken sich zugunsten der Gräser aus. Diese produzieren mit der in der Trockenzeit absterbenden oberirdischen Blattmasse überhaupt erst das nötige, leicht entzündliche Brennmaterial. Gleichzeitig schadet ihnen aufgrund ihrer typischen Wuchsform das Feuer weniger als den Holzpflanzen. Auch bei der Ausbreitung der $\mathrm{C}_{4}$-Gräser im Miozän haben Feuer wohl eine wichtige begünstigende Rolle gespielt.

\section{Evolution von Gräsern und Huftieren}

In den afrikanischen Savannen produzieren die Gräser und Gehölze eine große Menge an Biomasse, die von den zahlreichen großen Pflanzenfressern (Elefanten, Unpaar- und Paarhufer) und natürlich auch von Termiten verzehrt wird. Die für Gräser typische rasche Regeneration aus Knospen am oder im Boden kann als Anpassung an die Beweidung interpretiert werden. Auf der anderen Seite werden Fortbewegungsart und Extremitäten der afrikanischen großen Huftiere als Anpassung an die rasche Bewe-

Abb. 4 (oben): $\mathrm{C}_{4}$-Gräser in Kultur, Zea mays im Hintergrund und Panicum cf. maximum im Vordergrund.

Abb. 5 (Mitte): Periodisch in der Regenzeit überschwemmte Bereiche gehören auch zur Savanne. Im Hintergrund die Charakterart der afrikanischen Savanne, der Baobab (Adansonia digitata).

Abb. 6 (unten): Auch das findet sich in der Savanne: eine Natternzunge (Ophioglossum reticulatum), ein Vertreter der Farnpflanzen.

Abb. 7 (Seite 65): Die Dum-Palme (Hyphaene thebaica) ist in der westafrikanischen Savanne heimisch. Ganz ungewöhnlich für Palmen ist, dass sich ihr oberirdischer Stamm regelmäßig verzweigt. 
gung in offenen, waldfreien Lebensräumen, eben den Savannen, interpretiert. Parallel zur Ausbreitung der von $\mathrm{C}_{4}$-Gräsern dominierten Savannen im oberen Miozän (8-3 Mio. Jahre) sind neue Tiergemeinschaften entstanden, die sich durch zahlreiche große, pflanzenfressende Huftiere auszeichnen (z. B. Pferde, Nashörner, Antilopen, Elefanten). Das reiche Nahrungsangebot der Gräser ermöglichte die artenreiche Aufspaltung dieser Gruppen (Beerling \& Osborne 2006).

Schaut man sich die Vertreter von Gräsern und Huftieren noch genauer an, so fällt als Besonderheit bei den Gräsern die Einlagerung von Kieselsäure in die äußerste Zellschicht (Epidermis) auf. Es sind mikroskopisch kleine, oft hantelförmige Gebilde aus Kieselsäure, die eine Zelle fast ausfüllen und in regelmäßiger Verteilung über die Blattoberfläche hinweg vorkommen. Diese kleinen „Kieselsteine“ führen dazu, dass sich die Zähne der Grasfresser besonders stark abnutzen und die Nahrung schlechter aufgeschlossen werden kann. Bei vielen Savannengräsern finden sich die Kieselkörper in besonders hoher Konzentration. Schauen wir uns nun die Zähne der entsprechenden Weidetiere an, so finden wir dort besondere Anpassungen, um die Lebensdauer der überlebenswichtigen Kauorgane zu verlängern. Dies sind die hochkronigen (hypsodonten) Zähne, die besser an den Verschleiß beim Zerkauen der kieselsäurehaltigen Gräser angepasst sind. Dieser Zahntyp wird im Miozän bei Huftieren immer häufiger, zeitlich annähernd parallel zur Ausbreitung der $\mathrm{C}_{4}$-Gräser. Da Gräser (als Futter) und Weidetiere (als „Fraßfeinde“) in der Savanne interagieren, haben sie sich offensichtlich im Laufe der Evolution aneinander angepasst. Dazu gehört auf Seiten der Tiere auch die Anpassung von Körperbau, insbesondere des Fortbewegungsapparates, an die schnelle, effektive Fortbewegung in offener Vegetation. Einzelne Tiergruppen haben sich offenbar erst mit der Ausbreitung der Savannen besonders erfolgreich und artenreich entwickeln können, z. B. die Antilopen aus der Verwandtschaft der Paarhufer. Diese Form der Evolution, bei der verschiedene Arten, die in ihrem Lebensraum

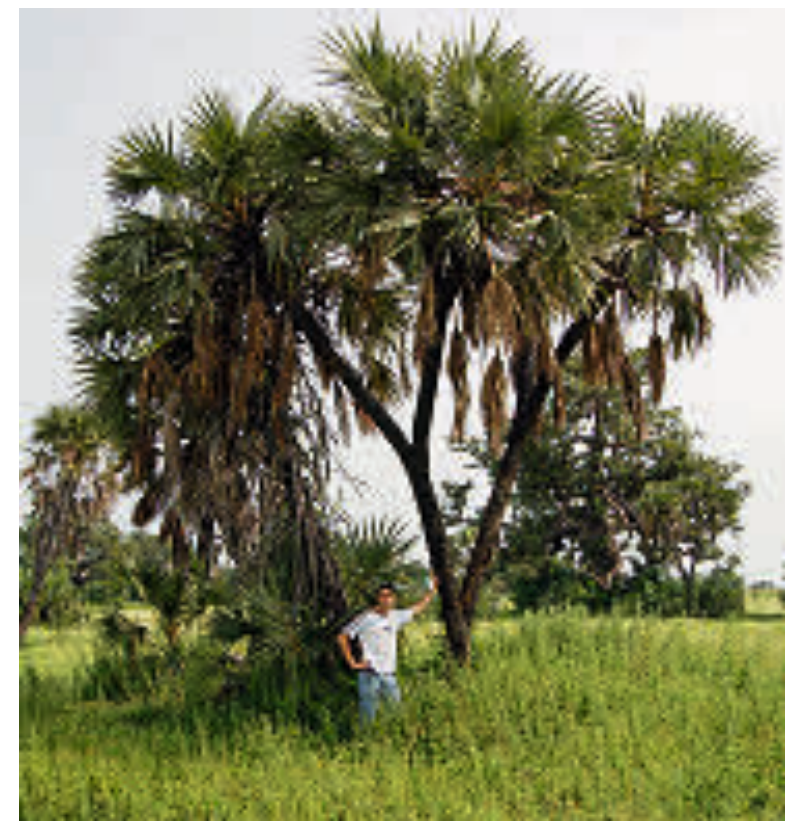

interagieren, wechselseitige Anpassungen aneinander zeigen, nennt man Coevolution. Gräser und große Herbivoren der afrikanischen Savanne sind ein in diesem Zusammenhang oft angeführtes Beispiel. Neueste Untersuchungen ergeben allerdings ein noch differenzierteres Bild: Die hochkronigen Zähne z. B. lassen sich nicht alleine zu Gräsern als Nahrung in Beziehung setzen, sondern offensichtlich wirken auch andere Faktoren mit wie allgemeine Trockenheit und Struktur der Vegetation.

\section{Wie werden sich die Savannen entwickeln?}

Savannen sind außerordentlich dynamische Lebensräume, bei denen Veränderungen der wichtigen Umweltfaktoren (z. B. Klima, Feuer, $\mathrm{CO}_{2}$-Konzentration in der Atmosphäre, menschlicher Eingriff) zum Verschieben des Verhältnisses von Gräsern und Gehölzen führt. In der Vergangenheit haben Klimaschwankungen mehrfach zu Verlagerungen der Vegetationszonen nach Norden bzw. Süden geführt.

Beobachtungen der jüngeren Vergangenheit, z. B. eines grüner Werdens von semiariden Gebieten („regreening of the Sahel“) und Vorhersagen zur Zukunft der Savannen anhand der Klimaszenarien für die nächsten hundert Jahre verblüffen mit Aussagen über eine stärkere Entwicklung der Gehölzschicht hin zu Trocken- 

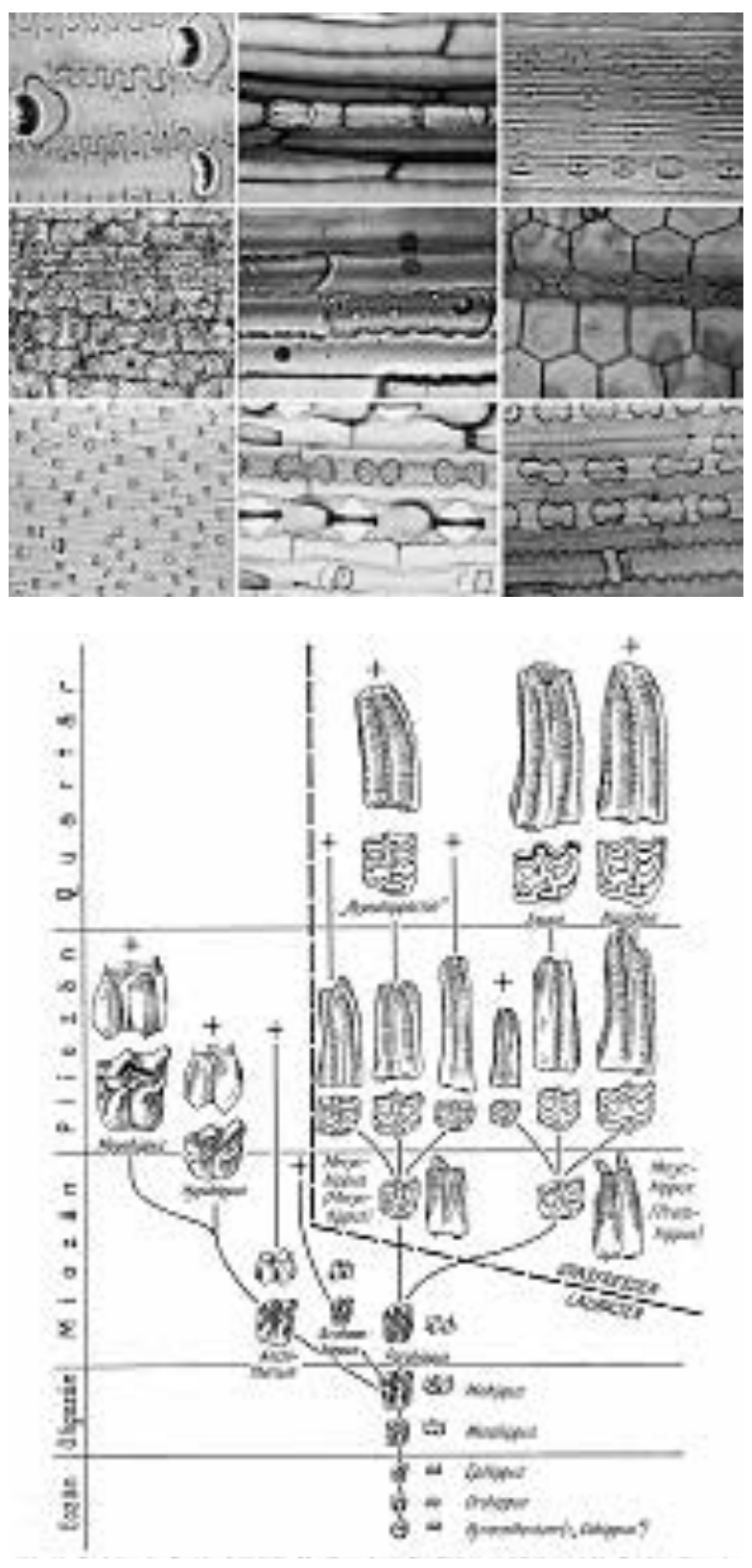

wäldern und Wäldern. Hintergrund ist die zu erwartende Zunahme des $\mathrm{CO}_{2}$-Gehaltes in der Luft, die sich wie eine Düngung auf das Wachstum der Gehölze auswirkt und ihnen einen Konkurrenzvorteil gegenüber den $\mathrm{C}_{4}$-Gräsern verschafft (s.o.). Ob die Zunahme der Gehölze aber wirklich eintritt, hängt vom alles dominierenden Faktor für die Zukunft der Savannen, dem Menschen, ab. Die Auswirkungen von Intensität und Form der Nutzung sowie die Frequenz der künstlichen Feuer werden die Folgen steigender $\mathrm{CO}_{2}$-Konzentratonen und die Interaktion mit immer weiter zurückgehenden Herden der Herbivoren wahrscheinlich bei Weitem überwiegen. Die Entwicklung der Savannen und ihrer biologischen Vielfalt wird also nicht von zukünftigen (co)evolutiven Errungenschaften abhängen, dazu finden die anthropogenen Veränderungen zu schnell statt. Es wird von unserem Verhalten und den Maßnahmen abhängen, die wir zur (wieder) nachhaltigen Nutzung der Savannen und zu ihrer Erhaltung ergreifen.

\section{Literatur}

Beerling, D. J. \& Osborne, C. P. 2006: The origin of the savanna biome. - Global Change Biology 12: 2023-2031. Bouchenak-Khelladi,Y. \& Hodkinson, T.R. 2011: Savanna biome evolution, climate change and the ecological expansion of $\mathrm{C}_{4}$ grasses. - In Hodkinson, T. R., Jones, M. B., Waldren, S. \& Parnell, J. A. N. (Hrsg.): Climate change, ecology and systematics: 156-174. - The systematics association special volume. - Cambridge. Edwards, E. J., Osborne, C. P., Strömberg, C. A. E., Sмiтh, S. A. \& C 4 Grasses Consortium 2010: The origins of $\mathrm{C}_{4}$ grasslands: Integrating evolutionary and ecosystem service. - Science 328: 587-591.

Franzen, J. L. 2007: Eozäne Equoidea (Mammalia, Perissodactyla) aus der Grube Messel bei Darmstadt (Deutschland): Funde der Jahre 1969-2000. - Schweizerische Paläontologische Abhandlungen 127: 1-245. Strömberg, C. A. E. 2011: Evolution of grasses and grassland ecosystems. - Ann. Rev. Earth Planet. Sci. 39: 517-544.

Thenius, E. \& Hofer, H. 1960: Stammesgeschichte der Säugetiere. - Berlin.

Watson, L. \& Dallwitz, M. J. 1992, onwards: The grass genera of the world: descriptions, illustrations, identification, and information retrieval; including synonyms, morphology, anatomy, physiology, phytochemistry, cytology, classification, pathogens, world and local distribution, and references. Version: 2nd April 2015. http://delta-intkey.com.

Abb. 8 (oben): Mikroskopische Aufnahmen der Epidermis verschiedener Gräser im Durchlicht. Neben den ganz unterschiedlich gestalteten Epidermiszellen und den Spaltöffnungen sind ebenfalls unterschiedlich geformte, meist hantelförmige Kieselkörper (Pfeile) gut zu erkennen. Sie füllen „ihre“ Zelle meist fast ganz aus (aus WATSON \& DALlWitz 1992 onwards).

Abb. 9 (unten): Die stammesgeschichtliche Entwicklung des Pferdegebisses im Laufe von 55 Millionen Jahren. Dargestellt ist je ein Oberkieferbackenzahn, gesehen von seiner Außenfläche und seiner Kaufläche. Man beachte die geradezu explosive Zunahme von Kronenhöhe und Verfältelung des Kauflächenmusters vor etwa 20-18 Millionen Jahren (aus Franzen 2007). 